LETTER TO THE EDITOR

\title{
Exact critical exponent for the shortest-path scaling function in percolation
}

\author{
Robert M Ziff \\ University of Michigan, Ann Arbor, MI 48109-2136, USA
}

Received 21 July 1999

\begin{abstract}
It is shown that the critical exponent $g_{1}$ related to pair-connectiveness and shortest-path (or chemical distance) scaling, recently studied by Porto et al, Dokholyan et al and Grassberger, can be found exactly in two dimensions by using a crossing-probability result of Cardy, with the outcome $g_{1}=25 / 24$. This prediction is consistent with existing simulation results.
\end{abstract}

\section{Introduction}

An important quantity describing percolation clusters is the chemical distance or shortest path [1]. There has been considerable effort studying its scaling properties for distances small compared with the size of the cluster (i.e., [2-4]) including recent work by Porto et al [5] and Dokholyan et al [6,7]. Very recently, Grassberger [8] has shown that these scaling properties can be analysed efficiently by studying the growth of two nearby clusters, a method first suggested in the work of Dokholyan et al [6]. In this note I show that a scaling relation for the growth of two clusters [6] can be combined with a previous result of Cardy [9] to find an exponent of the shortest-path behaviour exactly.

In [8], Grassberger considered the function $N(t)$ (which I call $N_{2}(t)$ ) giving the probability that two clusters grown from nearby seeds survive to at least time $t$, where clusters are grown by a Leath-type algorithm [10] and where $t$ is the number of generations or, equivalently, the chemical distance from the seeds to the growth sites. (To survive up to that time means that both clusters survive and remain distinct.) Another interpretation of $N_{2}(t)$ is that it gives the probability that two sites appear to belong to two different infinite clusters, when the environment is probed up to a chemical distance $t$ from the two sites. (As discussed in [8], two points a finite distance apart in fact belong to two different infinite clusters with probability zero.) At the critical point, $N_{2}(t)$ is presumed to behave as a power law

$$
N_{2}(t) \sim t^{-\mu}
$$

as $t \rightarrow \infty$. Grassberger also considered the probability $p(t)$ that the two clusters coalesce exactly at time $t ; p(t)$ is proportional to $\rho(\boldsymbol{x}-\boldsymbol{y}, 2 t)$, where $\boldsymbol{x}$ and $\boldsymbol{y}$ are the locations of the seed points and $\rho(\boldsymbol{x}, t)$ is the pair-connectiveness function, which behaves as $[11,12]$

$$
\rho(\boldsymbol{x}, t) \sim \frac{1}{t^{1+2 \beta / v_{t}}} \phi\left(r / t^{z}\right)
$$


in the scaling limit. The scaling function $\phi(\zeta)$ is presumed to behave as $\zeta^{g_{1}}$ for $\zeta \rightarrow 0$ [5], so $\rho(\boldsymbol{x}, t) \sim r^{g_{1}}$ for constant $t \gg r^{1 / z}$. Grassberger showed that these arguments imply $p(t) \sim t^{-\lambda}$ with

$$
\lambda=1+\frac{2 \beta}{v_{t}}+z g_{1}
$$

and furthermore argued that $\mu=\lambda-1$. The relation (3) was first given (in a slightly different notation) by Dokholyan et al [6].

Based upon an analogy to self-avoiding random walks, Porto et al [5] conjectured that $g_{1}$ is related to $d_{\min }=1 / z=v_{t} / v$ by

$$
g_{1}=d_{\min }-\beta / \nu \quad(\text { conjecture })
$$

which they found to be supported, to within the $\approx 5 \%$ error bars, by numerical measurements. This conjecture also appears in $[6,7]$. Inserting equation (4) into equation (3) implies

$$
\lambda=2+\frac{\beta}{v_{t}} \quad \text { (conjecture). }
$$

However, from precise simulations of $p(t)$ and $N_{2}(t)$, Grassberger found strong numerical evidence against the above conjecture (and provided theoretical arguments against it as well). He found, in two dimensions,

$$
\mu=1.1055(10) \quad \lambda=2.1055(10) \quad g_{1}=1.041(1)
$$

which are numerically inconsistent with the predictions $\mu=1.09213$ (5) and $g_{1}=1.0264(3)$ that follow from equations (4) and (5) and the known values $\beta=\frac{5}{36}, v=\frac{4}{3}$, and $d_{\min }=1.1306(3)$ [8], where numbers in parentheses following numerical data represent statistical errors in the last digit(s).

Here I show that $g_{1}$ can be found exactly by relating $N_{2}(t)$ to a crossing problem solved recently by Cardy [9]. Cardy has shown that for a rectangular system of dimensions $L_{v} \times L_{h}$, with periodic boundary conditions in the vertical direction, the probability of having at least $k$ clusters cross in the horizontal direction behaves, for large aspect ratio $R=L_{h} / L_{v}$, as

$$
P_{k}(R) \sim \mathrm{e}^{-a_{k} R}
$$

with $a_{1}=5 \pi / 24$ and $a_{k}=(2 \pi / 3)\left(k^{2}-1 / 4\right)$ for $k>1$. (The formula for $k=1$ is different because for one cluster it is not necessary to also have a crossing cluster on the dual lattice, while for $k>1$ crossing clusters there must be $k$ crossing dual-lattice clusters.) The probability that at least two clusters (or, to the same order, exactly two clusters) cross the rectangle is given by $P_{2} \sim \exp (-5 \pi R / 2)$.

Crossing problems in critical percolation are believed to be conformally invariant, because under a conformal transformation, in which all elements only expand or contract, the crossing properties of each element should remain unchanged $[13,14]$. One can transform the rectangle into an annulus by putting the four corners of the rectangle at $z=0,2 \pi R, 2 \pi R+2 \pi \mathrm{i}$ and $2 \pi \mathrm{i}$ on the complex- $z$ plane, and letting $z^{\prime}=\mathrm{e}^{z}$. The result on the $z^{\prime}$-plane is an annulus with an inner radius of 1 and an outer radius of $r=\mathrm{e}^{2 \pi R}$. The top and bottom edges of the rectangle close together, exactly matching the periodic boundary conditions. Assuming conformal invariance of the crossing probability, it follows from (7) that the probability $p_{k}$ that at least $k$ clusters cross between the inner and outer boundaries of the annulus is given by

$$
p_{k}(r) \sim r^{-a_{k} /(2 \pi)}
$$

or $p_{1}(r) \sim r^{-5 / 48}, p_{2}(r) \sim r^{-5 / 4}, p_{3}(r) \sim r^{-35 / 12}$, etc. Now, one can associate $p_{2}(r)$ with the quantity $N_{2}(t)$ of equation (1) by transforming from chemical distance $t$ to the radial distance $r$ using $r \sim t^{z}$. This yields

$$
N_{2}(t) \sim t^{-5 z / 4}
$$


which implies by (1)

and by (3) gives

$$
\mu=\frac{5 z}{4}=\frac{5 v_{t}}{4 v}=1.1056(3)
$$

$$
g_{1}=\frac{5}{4}-\frac{2 \beta}{v}=\frac{25}{24}=1.041666 \ldots
$$

These predictions are consistent with Grassberger's measurements (6) as well as Porto et al's determination $g_{1}=1.04(5)$. However, (11) is apparently inconsistent with the conjecture (4), since it would imply

$$
d_{\min }=\frac{5}{4}-\frac{\beta}{v}=\frac{55}{48}=1.1458333 \ldots \quad \text { (conjecture) }
$$

which differs from the measured values of $d_{\min }, 1.1306(3)$ [11] and 1.130(4) [15].

Another way of looking at Grassberger's numerical results is that they serve to confirm the ideas of conformal invariance and Cardy's formula for $k=2$ to high precision. Note that Cardy's formula for $k=1,2$ and 3 has also been verified numerically by Shchur and Kosyakov [16-18]. Indeed, equation (9) can be generalized for the probability $N_{k}(t)$ that $k$ clusters remain alive and distinct up to time $t$,

$$
N_{k}(t) \sim p_{k}\left(t^{z}\right) \sim t^{-z a_{k} /(2 \pi)}
$$

so that $N_{3}(t) \sim t^{-35 z / 12} \sim t^{-2.580}$, etc. Grassberger has also measured this quantity for $k=3$ and $k=4$, and the behaviour he finds is consistent with the above predictions [19].

I note, finally, that the relation $p_{1}(r) \sim r^{-5 / 48}$ following (8) is just the statement that the probability a cluster grows to a radius greater or equal than $r$ is $p_{1}(r) \sim r^{D-d}$. The latter formula follows from $P \geqslant s=\int_{s}^{\infty} s n_{s} \sim s^{2-\tau}$ with $s=r^{D}$ with $D=\frac{91}{48}$, and the hyperscaling relation $\tau-1=d / D$. Transforming from the annulus to a rectangle yields Cardy's result (7) for $k=1, P_{1}(R) \sim \mathrm{e}^{-2 \pi(d-D) R}$ (valid for $d=2$ only).

In conclusion, I have shown that the density of growth sites on two-dimensional percolation clusters behaves as $r^{25 / 24}$ for large time and small $r$.

The author thanks P Grassberger, J Cardy and S Havlin for comments. This material is based upon work supported by the US National Science Foundation Grant No DMR-9520700.

\section{References}

[1] Havlin S and Ben-Avraham D 1987 Adv. Phys. 36695

[2] Barma M and Ray P 1986 Phys. Rev. B 343403

[3] Bunde A, Roman H E, Russ S, Aharony A and Harris A B 1992 Phys. Rev. Lett. 693189

[4] Roman H E 1995 Phys. Rev. E 515422

[5] Porto M, Havlin S, Roman H E and Bunde A 1998 Phys. Rev. E 58 R5205

[6] Dokholyan N V, Lee Y, Buldyrev S V, Havlin S, King P R and Stanley H E 1998 J. Stat. Phys. 93603

[7] Dokholyan N V, Buldyrev S V, Havlin S, King P R, Lee Y and Stanley H E 1999 Physica A 26655

[8] Grassberger P 1999 J. Phys. A: Math. Gen. 326233

[9] Cardy J 1998 J. Phys. A: Math. Gen. 31 L105

[10] Leath P L 1976 Phys. Rev. B 145046

[11] Grassberger P 1983 Math. Biosci. 62157

[12] Grassberger P 1992 J. Phys. A: Math. Gen. 255867

[13] Aizenman M 1995 STATPHYS 19, Proc. (Xiamen, 1995) ed H Bai-lin (Singapore: World Scientific)

[14] Langlands R P, Pouliot Ph and Saint-Aubin Y 1994 Bull. Am. Math. Soc. 301

[15] Herrmann H J and Stanley H E 1988 J. Phys. A: Math. Gen. 21 L829

[16] Shchur L N and Kosyakov S S 1997 Int. J. Mod. Phys. C 8607

[17] Shchur L N and Kosyakov S S 1998 Nucl. Phys. B (Proc. Suppl.) A-C 63664

[18] Shchur L N 1999 Proc. 12th Annual Workshop on Computer Simulation Studies in Condensed Matter Physics (Athens, GA) Preprint cond-mat/9906013

[19] Grassberger P Private communication 